\title{
How to extract physics from HBT radius parameters
}

\author{
Ulrich Heinz* \\ Physics Department, Duke University, Durham, NC 27708-0305, USA
}

I review recent progress in the understanding of the connection between the space-time structure of the particle emitting source and the form of the two-particle correlation function in momentum space. Based on a new scheme for calculating the HBT radius parameters from the emission function, strategies are suggested to separate for rapidly expanding sources the information on the spatial and temporal structure of the source. To this end a new fitting function for the two-particle correlation function is proposed. Its usefulness is demonstrated for a typical expanding model source, and it is shown how the dependence of the resulting fit parameters on the momentum of the particle pair can be used to measure the longitudinal and transverse expansion of the source.

\section{INTRODUCTION}

The only known way to obtain direct experimental information on the space-time structure of the particle emitting source created in a relativistic nuclear collision is through two-particle intensity (Hanbury-Brown-Twiss (HBT)) interferometry [ [1]. The goal of this method is to extract the space-time structure of the source from momentum spectra which are the only measurable quantities, making use of the quantum statistical correlations between pairs of identical particles. This information is crucial for an assessment of theoretical models which try to extract the energy density of the source from the measured single particle spectra and particle multiplicity densities in momentum space. Reliable estimates of the source volume and the energy density are, on the other hand, indispensable for an experimental proof that high energy collisions can successfully generate large volumes of matter with extreme energy density, where a transition into deconfined quark matter might be possible.

For many years HBT interferometry of hadron-hadron and nucleus-nucleus collisions was motivated by the naive expectation, based on the experience with photon interferometry for stars, that the width of the two-particle correlation function in the relative momentum directly measures the geometric size of the source. This expectation is wrong. Unlike stars in the universe, the sources created in hadronic or heavy-ion collisions may feature inhomogeneous temperature profiles and strong collective dynamical expansion. We now know [2, 3, 4, 5, 6] that for such sources the HBT radius parameters generally don't measure the full source size, but only so-called "space-time regions of homogeneity"

*On sabbatical leave from Institut für Theoretische Physik, Universität Regensburg, D-93040 Regensburg, Germany. - Invited talk given at Quark Matter '96, Heidelberg, 20.-24.5.96 


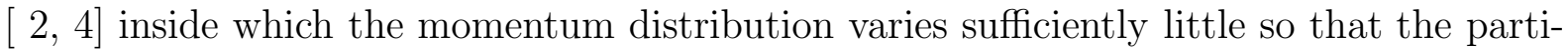
cles can actually show the quantum statistical correlations. The size of these homogeneity regions varies with the momentum of the emitted particles, causing a dependence of the HBT parameters on the pair momentum [ 2, 3, 4, 5, 6, 17, 8, 9, 10, 11, 12, 13, 14]. The detailed momentum dependence is, however, model-dependent, and in general it is not simple [12]. The extraction of the strength of the collective flow from HBT data is further complicated by significant resonance decay contributions which also induce a momentum dependence of the HBT radius parameters and of the so-called "incoherence parameter" [10, 15].

The finite lifetime of the sources created in nuclear collisions leads to another complication: the HBT radius parameters generally mix the spatial and temporal aspects of the source extension in a non-trivial and reference frame dependent way, in particular if at the same time the source undergoes collective expansion. The origin and pattern of this mixing was clarified in a recent series of publications from the Regensburg group [ 6, 11, 12, 13, 14. Two major new results have resulted from this work: (i) the discovery [6] of a new cross term in the two-particle correlation function mixing the outward and longitudinal components of the relative momentum between the two particles, and (ii) the new YKP (Yano-Koonin-Podgoretskiı) parametrisation of the correlation function [ 11, 13, 14. The latter permits the experimental determination of the longitudinal velocity of the source volume element where most of the particle pairs originate (as a function of the pair momentum) and achieves a nearly perfect factorization of the longitudinal, transverse and temporal homogeneity regions of the source (again as functions of the pair momentum) in the source rest frame. Furthermore it provides for a clean separation of the longitudinal and transverse dynamics of the source.

In this talk I review these new theoretical developments and exemplify them for a class of simple model emission functions for thermalized sources with collective transverse and longitudinal expansion and finite space-time geometry.

\section{EMISSION FUNCTION AND PARTICLE SPECTRA}

The single and two particle spectra are defined as

$$
\begin{aligned}
P_{1}(\mathbf{p}) & =E_{p} \frac{d N}{d^{3} p}=E_{p}\left\langle\hat{a}_{p}^{+} \hat{a}_{p}\right\rangle \\
P_{2}\left(\mathbf{p}_{\mathbf{1}}, \mathbf{p}_{\mathbf{2}}\right) & =E_{1} E_{2} \frac{d N}{d^{3} p_{1} d^{3} p_{2}}=E_{1} E_{2}\left\langle\hat{a}_{p_{1}}^{+} \hat{a}_{p_{2}}^{+} \hat{a}_{p_{2}} \hat{a}_{p_{1}}\right\rangle
\end{aligned}
$$

in terms of creation and destruction operators for on-shell particles with momenta $\mathbf{p}_{i}$, where $\langle\ldots\rangle$ denotes an average over the source ensemble. $P_{1}$ and $P_{2}$ are normalized to the average number of particles $\langle N\rangle$ and pairs $\langle N(N-1)\rangle$ per event, respectively. The two-particle correlation function is defined as

$C\left(\mathbf{p}_{\mathbf{1}}, \mathbf{p}_{\mathbf{2}}\right)=\frac{\langle N\rangle^{2}}{\langle N(N-1)\rangle} \frac{P_{2}\left(\mathbf{p}_{\mathbf{1}}, \mathbf{p}_{\mathbf{2}}\right)}{P_{1}\left(\mathbf{p}_{\mathbf{1}}\right) P_{1}\left(\mathbf{p}_{\mathbf{2}}\right)}$.

For uncorrelated emission and in the absence of final state interactions [16] one can prove

[17, 18] a generalized Wick theorem for the factorisation of the 2-particle spectrum (2) 
and obtains

$C(\mathbf{q}, \mathbf{K})=1 \pm \frac{\left|\left\langle\hat{a}_{p_{1}}^{+} \hat{a}_{p_{2}}\right\rangle\right|^{2}}{\left\langle\hat{a}_{p_{1}}^{+} \hat{a}_{p_{1}}\right\rangle\left\langle\hat{a}_{p_{2}}^{+} \hat{a}_{p_{2}}\right\rangle}$

where $\mathbf{q}=\mathbf{p}_{\mathbf{1}}-\mathbf{p}_{\mathbf{2}}$ and $\mathbf{K}=\left(\mathbf{p}_{\mathbf{1}}+\mathbf{p}_{\mathbf{2}}\right) / 2$ denote the relative and total momentum of the particle pair, and the positive (negative) sign applies for bosons (fermions). Note that the second term is positive definite.

These expressions can be further simplified and turned into a practical starting point for computations by introducing the emission function $S(x, K)$. It is defined in terms of the classical source amplitude $J(x)$ for creating a free pion state [ 17] via the Wigner transform of its associated density matrix

$S(x, K)=\int \frac{d^{4} y}{2(2 \pi)^{3}} e^{-K \cdot y}\left\langle J^{*}\left(x+\frac{y}{2}\right) J\left(x-\frac{y}{2}\right)\right\rangle$

and is the quantum mechanical analogue of the classical phase-space density which gives the probability for creating a free particle with four-momentum $K$ at space-time point $x$. In terms of this emission function the single particle spectrum is given by

$E_{K} \frac{d N}{d^{3} K}=\int d^{4} x S(x, K)$

where the r.h.s. is to be evaluated on-shell, i.e. at $K^{0}=E_{K}=\sqrt{m^{2}+\mathbf{K}^{2}}$. The twoparticle correlation function is obtained from [ 19, 17, 7, 18]

$C(\mathbf{q}, \mathbf{K}) \approx 1 \pm \frac{\left|\int d^{4} x S(x, K) e^{i q \cdot x}\right|^{2}}{\left|\int d^{4} x S(x, K)\right|^{2}}=1 \pm\left|\left\langle e^{i q \cdot x}\right\rangle\right|^{2}$

where the r.h.s. must be evaluated at $q=p_{1}-p_{2}, K=\left(p_{1}+p_{2}\right) / 2$ with $p_{i}$ on-shell. (This implies $K \cdot q=0$.) The approximation consists of replacing the single particle spectra at $p_{1}$ and $p_{2}$ in the denominator by the spectrum at $K=\left(p_{1}+p_{2}\right) / 2$; it is exact for exponential momentum spectra and a good approximation in practice [ 6]. The second equality in (7) defines a ( $K$-dependent) average over the emission function of which we will make abundant use below. A useful feature is that in (7) the emission function can be to very good approximation [ 6, 9] evaluated at $K^{0}=E_{K}$, i.e. on the classical energy shell, since the typical source radii are larger than the Compton wavelengths of the observed hadrons. This warrants the replacement of the Wigner density $S(x, K)$ by a classical phase-space distribution in practical calculations.

Due to the on-shell constraint $q \cdot K=0$ the four components of $q$ are not independent, but related by

$q^{0}=\boldsymbol{\beta} \cdot \mathbf{q} \quad$ with $\quad \boldsymbol{\beta}=\frac{\mathbf{K}}{K^{0}} \approx \frac{\mathbf{K}}{E_{K}}$.

The Fourier transform in (7) is therefore not invertible, and the reconstruction of the space-time structure of the source from HBT measurements will thus always require additional model assumptions. Furthermore, inserting (8) into (7),$i q \cdot x=i \mathbf{q} \cdot(\mathbf{x}-\boldsymbol{\beta} t)$, we see that the correlator $C(\mathbf{q}, \mathbf{K})$ mixes the spatial and temporal information in a non-trivial 
way which depends on the pair velocity $\boldsymbol{\beta}$. Only for time-independent sources things become simple: the correlator then measures the Fourier transform of the spatial source distribution, however only in the directions perpendicular to $\boldsymbol{\beta}$ since the time integration leads to a $\delta$-function $\delta(\boldsymbol{\beta} \cdot \mathbf{q})$.

From Eq. (7) it is clear that, unless the emission function factorizes in $x$ and $K$, $S(x, K)=F(x) G(K)$ (in which case $G(K)$ cancels between numerator and denominator), the correlator is a function of both $\mathbf{q}$ and $\mathbf{K}$. If one parametrises it by a Gaussian in $q$ (see Sec. (4) this results in $\mathbf{K}$-dependent width parameters ("HBT radii"). In thermal sources $x-K$ correlations which spoil such a factorization can be induced by temperature gradients and/or collective expansion (with a 4-velocity $u^{\mu}(x)$ ): in both cases the momentum spectrum $\sim \exp [-p \cdot u(x) / T(x)]$ of the emitted particles depends on the emission point.

\section{MODEL-INDEPENDENT EXPRESSIONS FOR THE HBT RADII}

One of the crucial questions is, of course, to what extent a measured $\mathbf{K}$-dependence of the HBT radii allows for a quantitative reconstruction of the collective source dynamics. To answer it we must first learn more about the physical meaning of these "radii". To this end it is useful to write the emission function in the following form [11, 12, 13]:

$S(x, K)=N(\mathbf{K}) S(\bar{x}(\mathbf{K}), K) \exp \left[-\frac{1}{2} \tilde{x}^{\mu}(\mathbf{K}) B_{\mu \nu}(\mathbf{K}) \tilde{x}^{\nu}(\mathbf{K})\right]+\delta S(x, K)$,

where (with expectation values defined as in (7))

$\bar{x}_{\mu}(\mathbf{K})=\left\langle x_{\mu}\right\rangle, \quad \tilde{x}_{\mu}(\mathbf{K})=x_{\mu}-\bar{x}_{\mu}(\mathbf{K}), \quad\left(B^{-1}\right)_{\mu \nu}(\mathbf{K})=\left\langle\tilde{x}_{\mu} \tilde{x}_{\nu}\right\rangle$.

This construction ensures that the term $\delta S$ has vanishing zeroth, first and second order moments and thus contains only higher order information on sharp edges, wiggles, secondary peaks, etc. in the source. It was shown numerically [12] to have negligible influence on the half width of the correlation function and to contribute only weak, essentially unmeasurable structures in $C(\mathbf{K}, \mathbf{q})$ at large values of $\mathbf{q}$. Neglecting $\delta S$, the two-particle correlation function (7) can be calculated analytically:

$C(\mathbf{K}, \mathbf{q})=1+\exp \left[-q^{\mu} q^{\nu}\left\langle\tilde{x}_{\mu} \tilde{x}_{\nu}\right\rangle(\mathbf{K})\right]$.

Please note that the point $\bar{x}^{\mu}(\mathbf{K})$ of maximum emissivity at momentum $\mathbf{K}$ is unmeasurable [ 13, 20]. Only the $\mathbf{K}$-dependent effective widths ("lengths of homogeneity") $\left\langle\tilde{x}_{\mu} \tilde{x}_{\nu}\right\rangle(\mathbf{K})$ of the source of particles with momentum $\mathbf{K}$ are accessible by HBT interferometry.

Actually, due to the on-shell constraint (8), only 6 linear combinations of the 10 variances $\left\langle\tilde{x}_{\mu} \tilde{x}_{\nu}\right\rangle(\mathbf{K})$ are measurable [ 11]; in the case of azimuthal symmetry of the source around the beam axis, this number reduces to 4 out of 7 . Which linear combinations occur in practice depends on the way the correlation function is parametrised. The general form (11) together with (8) still provide some freedom as to which components of $q$ to keep as independent variables (see Sec. 1). But whichever choice one makes, all the $\mathbf{K}$ dependent parameters ("HBT radii") in the resulting Gaussian function of $q$ can be easily calculated from the variances $\left\langle\tilde{x}^{\mu} \tilde{x}^{\nu}\right\rangle$, i.e. by simple quadrature formulae, for arbitrary emission functions $S(x, K)$. The relation between the HBT parameters and the variances is model-independent, i.e. it does not depend on the form of the emission function $S(x, K)$. 


\section{STANDARD AND YKP FITS TO THE CORRELATION FUNCTION}

For the following discussion we employ the conventional [ 7, 8] Cartesian coordinate system with $z$ along the beam axis and $\mathbf{K}$ lying in the $x$-z-plane. The $z$-component of a 3 -vector is labelled by $l$ (for longitudinal), the $x$-component by $o$ (for outward) and the $y$-component by $s$ (for sideward). Then $\beta_{s}=0$ such that $q^{0}=\beta_{\perp} q_{o}+\beta_{l} q_{l}$, with $\beta_{\perp}=\left|\mathbf{K}_{\perp}\right| / K^{0}$ being (approximately) the velocity of the particle pair transverse to the beam direction while $\beta_{l}$ is its longitudinal component.

The standard Cartesian parametrization [ [6] of the correlation function is obtained by using this condition to eliminate $q^{0}$ from Eq. (11). One obtains

$C(\mathbf{K}, \mathbf{q})=1+\exp \left[-\sum_{i, j=s, o, l} R_{i j}^{2}(\mathbf{K}) q_{i} q_{j}\right]$

where the $6 \mathrm{HBT}$ "radius parameters" $R_{i j}$ are given as [ 6, 21]

$R_{i j}^{2}(\mathbf{K})=\left\langle\left(\tilde{x}_{i}-\beta_{i} \tilde{t}\right)\left(\tilde{x}_{j}-\beta_{j} \tilde{t}\right)\right\rangle, \quad i, j=s, o, l$,

through through the space-time variances of the source. For an azimuthally symmetric sample of collision events, $C(\mathbf{q}, \mathbf{K})$ is symmetric with respect to $q_{s} \rightarrow-q_{s}$ [ 11]. Then $R_{o s}^{2}=R_{s l}^{2}=0$ and

$$
\begin{aligned}
C(\mathbf{K}, \mathbf{q}) & =1+\exp \left[-R_{s}^{2}(\mathbf{K}) q_{s}^{2}-R_{o}^{2}(\mathbf{K}) q_{o}^{2}-R_{l}^{2}(\mathbf{K}) q_{l}^{2}-2 R_{o l}^{2}(\mathbf{K}) q_{o} q_{l}\right], \quad \text { with } \\
R_{s}^{2}(\mathbf{K}) & =\left\langle\tilde{y}^{2}\right\rangle \\
R_{o}^{2}(\mathbf{K}) & =\left\langle\left(\tilde{x}-\beta_{\perp} \tilde{t}\right)^{2}\right\rangle \\
R_{l}^{2}(\mathbf{K}) & =\left\langle\left(\tilde{z}-\beta_{l} \tilde{t}\right)^{2}\right\rangle \\
R_{o l}^{2}(\mathbf{K}) & =\left\langle\left(\tilde{x}-\beta_{\perp} \tilde{t}\right)\left(\tilde{z}-\beta_{l} \tilde{t}\right)\right\rangle .
\end{aligned}
$$

The cross-term (18) was only recently discovered [ 6]. Clearly these HBT radius parameters mix spatial and temporal information on the source in a non-trivial way. Their interpretation in various reference systems, in particular the meaning of the generally non-vanishing cross-term $R_{o l}^{2}$, was extensively discussed in Refs. [ 6, 11, 12], by analysing these expressions analytically for a large class of (azimuthally symmetric) model source functions and comparing with the numerically calculated correlation function (7). An important observation resulting from these studies is that the difference

$R_{\text {diff }}^{2} \equiv R_{o}^{2}-R_{s}^{2}=\beta_{\perp}^{2}\left\langle\tilde{t}^{2}\right\rangle-2 \beta_{\perp}\langle\tilde{x} \tilde{t}\rangle+\left(\left\langle\tilde{x}^{2}\right\rangle-\left\langle\tilde{y}^{2}\right\rangle\right)$

is generally dominated by the first term on the r.h.s. [14] and thus provides access to the lifetime $\Delta t=\sqrt{\left\langle t^{2}\right\rangle-\langle t\rangle^{2}}$ of the source [22] (more exactly: the duration of the particle emission process). In heavy-ion collisions, due to rapid expansion of the source, one would generally not expect $\left\langle\tilde{t}^{2}\right\rangle$ to be much larger than either $\left\langle\tilde{x}^{2}\right\rangle$ or $\left\langle\tilde{y}^{2}\right\rangle$ (see however [23] for possible exceptions near a phase transition to QGP). In the standard fit one is not sensitive to small values of $\Delta t$ since Eq. (19) then involves a small difference of two large numbers, each associated with standard experimental errors. The factor $\beta_{\perp}^{2} \leq 1$ in front of $\left\langle\tilde{t}^{2}\right\rangle$ further complicates its extraction, in particular at low $K_{\perp}$ where $\Delta t(\mathbf{K})$ is usually largest (see below). 
This problem is avoided in the Yano-Koonin-Podgoretskil parametrisation [ 24, 25, 11, 13, 14] of the correlation function for azimuthally symmetric systems. It is based on an elimination of $q_{o}$ and $q_{s}$ in terms of $q_{\perp}^{2}=q_{o}^{2}+q_{s}^{2}, q^{0}$, and $q_{3}$ in (11):

$$
C(\mathbf{q}, \mathbf{K})=1+\exp \left[-R_{\perp}^{2} q_{\perp}^{2}-R_{\|}^{2}\left(q_{l}^{2}-\left(q^{0}\right)^{2}\right)-\left(R_{0}^{2}+R_{\|}^{2}\right)(q \cdot U)^{2}\right]
$$

with four $\mathbf{K}$-dependent parameters $R_{\perp}, R_{\|}, R_{0}$, and $U^{\mu}$ where the latter is a 4 -velocity with only a longitudinal spatial component:

$U(\mathbf{K})=\gamma(\mathbf{K})(1,0,0, v(\mathbf{K}))$, with $\gamma=\left(1-v^{2}\right)^{-1 / 2}$

This parametrisation has the advantage that the fitted YKP parameters $R_{\perp}^{2}(\mathbf{K}), R_{\|}^{2}(\mathbf{K})$, and $R_{0}^{2}(\mathbf{K})$ do not depend on the longitudinal velocity of the observer system. They (as well as $v(\mathbf{K})$ ) can be calculated from the variances $\left\langle\tilde{x}^{\mu} \tilde{x}^{\nu}\right\rangle$ in any reference frame (see [ 13] for explicit expressions), but their physical interpretation is easiest in terms of coordinates measured in the frame where $v(\mathbf{K})$ vanishes. There they are given by [11]

$$
\begin{aligned}
R_{\perp}^{2}(\mathbf{K}) & =R_{s}^{2}(\mathbf{K})=\left\langle\tilde{y}^{2}\right\rangle, \\
R_{\|}^{2}(\mathbf{K}) & =\left\langle\left(\tilde{z}-\beta_{l} \tilde{x} / \beta_{\perp}\right)^{2}\right\rangle-\beta_{l}^{2}\left\langle\tilde{y}^{2}\right\rangle / \beta_{\perp}^{2} \approx\left\langle\tilde{z}^{2}\right\rangle, \\
R_{0}^{2}(\mathbf{K}) & =\left\langle\left(\tilde{t}-\tilde{x} / \beta_{\perp}\right)^{2}\right\rangle-\left\langle\tilde{y}^{2}\right\rangle / \beta_{\perp}^{2} \approx\left\langle\tilde{t}^{2}\right\rangle,
\end{aligned}
$$

where in the last two expressions the approximation consists of dropping generically small [11] terms (for a quantitative discussion see [14]). The first expression (22] remains true in any longitudinally boosted frame.

Eq. (24) shows that the YKP parameter $R_{0}(\mathbf{K})$ measures directly (up to the neglected small terms) the time duration $\Delta t(\mathbf{K})$ during which particles of momentum $\mathbf{K}$ are emitted, in the frame were the YKP velocity $v(\mathbf{K})=0$. The advantage compared to the standard Cartesian fit is that here it is fitted directly, and no problems of differences of large numbers occur in its extraction.

Since the standard Cartesian and YKP parametrizations (14) and (20) of the correlator differ only by the choice of independent components of $q$, the two sets of HBT parameters must be related. One finds [ 13]

$$
\begin{aligned}
R_{s}^{2} & =R_{\perp}^{2} \\
R_{\text {diff }}^{2} & =R_{o}^{2}-R_{s}^{2}=\beta_{\perp}^{2} \gamma^{2}\left(R_{0}^{2}+v^{2} R_{\|}^{2}\right) \\
R_{l}^{2} & =\left(1-\beta_{l}^{2}\right) R_{\|}^{2}+\gamma^{2}\left(\beta_{l}-v\right)^{2}\left(R_{0}^{2}+R_{\|}^{2}\right) \\
R_{o l}^{2} & =\beta_{\perp}\left(-\beta_{l} R_{\|}^{2}+\gamma^{2}\left(\beta_{l}-v\right)^{2}\left(R_{0}^{2}+R_{\|}^{2}\right)\right) .
\end{aligned}
$$

These relations provide a powerful consistency check on the experimental fitting procedure of the correlation function, of similar value as the relation [ 11, 12] $\lim _{K_{\perp} \rightarrow 0}\left(R_{o}(\mathbf{K})-\right.$ $\left.R_{s}(\mathbf{K})\right)=0$ which results from azimuthal symmetry. 


\section{A SIMPLE SOURCE MODEL}

For a quantitative discussion of the physical behaviour of the HBT radius parameters, in particular of their $\mathbf{K}$-dependence, we use a simple model for the emission function of a finite expanding thermalized source [11]:

$S(x, K)=\frac{M_{\perp} \cosh (\eta-Y)}{(2 \pi)^{3} \sqrt{2 \pi(\Delta \tau)^{2}}} \exp \left[-\frac{K \cdot u(x)}{T}-\frac{\left(\tau-\tau_{0}\right)^{2}}{2(\Delta \tau)^{2}}-\frac{r^{2}}{2 R^{2}}-\frac{\eta-\eta_{0}^{2}}{2(\Delta \eta)^{2}}\right]$.

Here $r=\sqrt{x^{2}+y^{2}}$, the spacetime rapidity $\eta=\frac{1}{2} \ln [(t+z) /(t-z)]$ and the longitudinal proper time $\tau=\sqrt{t^{2}-z^{2}}$ parametrize the spacetime coordinates $x^{\mu}$, with measure $d^{4} x=\tau d \tau d \eta r d r d \phi . Y=\frac{1}{2} \ln \left[\left(1+\beta_{l}\right) /\left(1-\beta_{l}\right)\right]$ and $M_{\perp}=\sqrt{m^{2}+K_{\perp}^{2}}$ parametrise the longitudinal and transverse components of the pair momentum $\mathbf{K}$.
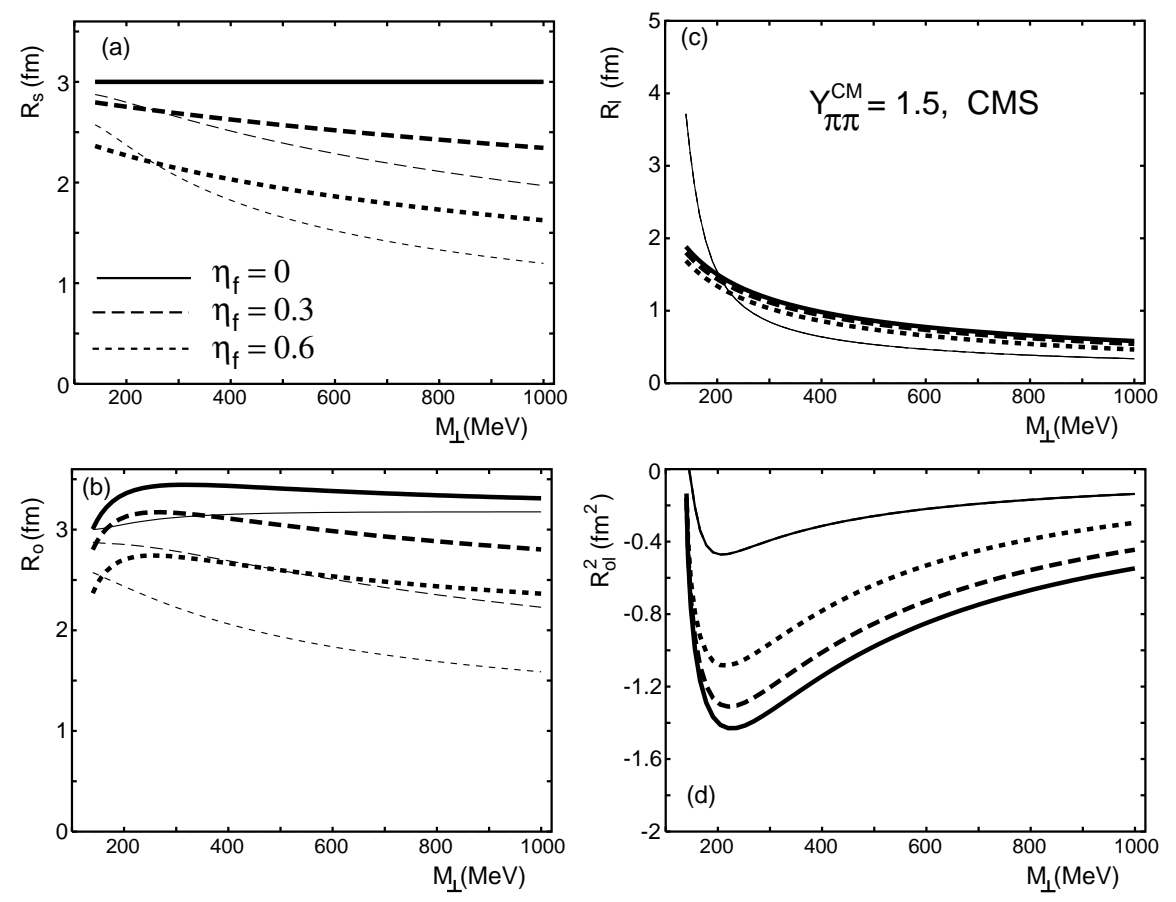

Fig.1. The standard Cartesian parameters $R_{s}$ (a), $R_{o}$ (b), $R_{l}$ (c), and $R_{o l}^{2}(\mathrm{~d})$ in the CMS for pion pairs with c.m. rapidity $Y=1.5$, as functions of $M_{\perp}$ for 3 different values for the transverse flow $\eta_{f}$. The thick lines are exact numerical results from Eqs. (15-18), the thin lines are obtained from the analytical approximations given in Ref. [5]. (Figure taken from Ref. [27].)

$T$ is the freeze-out temperature, $R$ is the transverse geometric (Gaussian) radius of the source, $\tau_{0}$ its average freeze-out proper time, $\Delta \tau$ the mean proper time duration of particle emission, and $\Delta \eta$ parametrises the finite longitudinal extension of the source. The expansion flow velocity $u^{\mu}(x)$ is parametrised as

$u^{\mu}(x)=\left(\cosh \eta_{l} \cosh \eta_{t}(r), \sinh \eta_{t}(r) \mathbf{e}_{r}, \sinh \eta_{l} \cosh \eta_{t}(r)\right), \eta_{l}=\eta, \eta_{t}(r)=\eta_{f}(r / R)$ 
with a boost-invariant longitudinal flow rapidity and a linear transverse flow rapidity profile. $\eta_{f}$ scales the strength of the transverse flow. The scalar product in the exponent of the Boltzmann factor can then be written as

$K \cdot u(x)=M_{\perp} \cosh (\eta-Y) \cosh \eta_{t}(r)-K_{\perp} \frac{x}{r} \sinh \eta_{t}(r)$.

Please note that for non-zero transverse momentum $K_{\perp}$, a finite transverse flow breaks the azimuthal symmetry of the emission function via the second term in (31). For $\eta_{f}=0$ the source has no explicit $K_{\perp}$-dependence, and $M_{\perp}$ is the only relevant scale. As will be discussed in Sec. 6.3 this gives rise to perfect $M_{\perp}$-scaling of the YKP radius parameters in the absence of transverse flow, which is again broken for non-zero transverse flow [26].

For the numerical calculations below we have selected one fixed set of source parameters: $R=3 \mathrm{fm}, \tau_{0}=3 \mathrm{fm} / c, \Delta \tau=1 \mathrm{fm} / c, \Delta \eta=1.2, T=140 \mathrm{MeV}$.
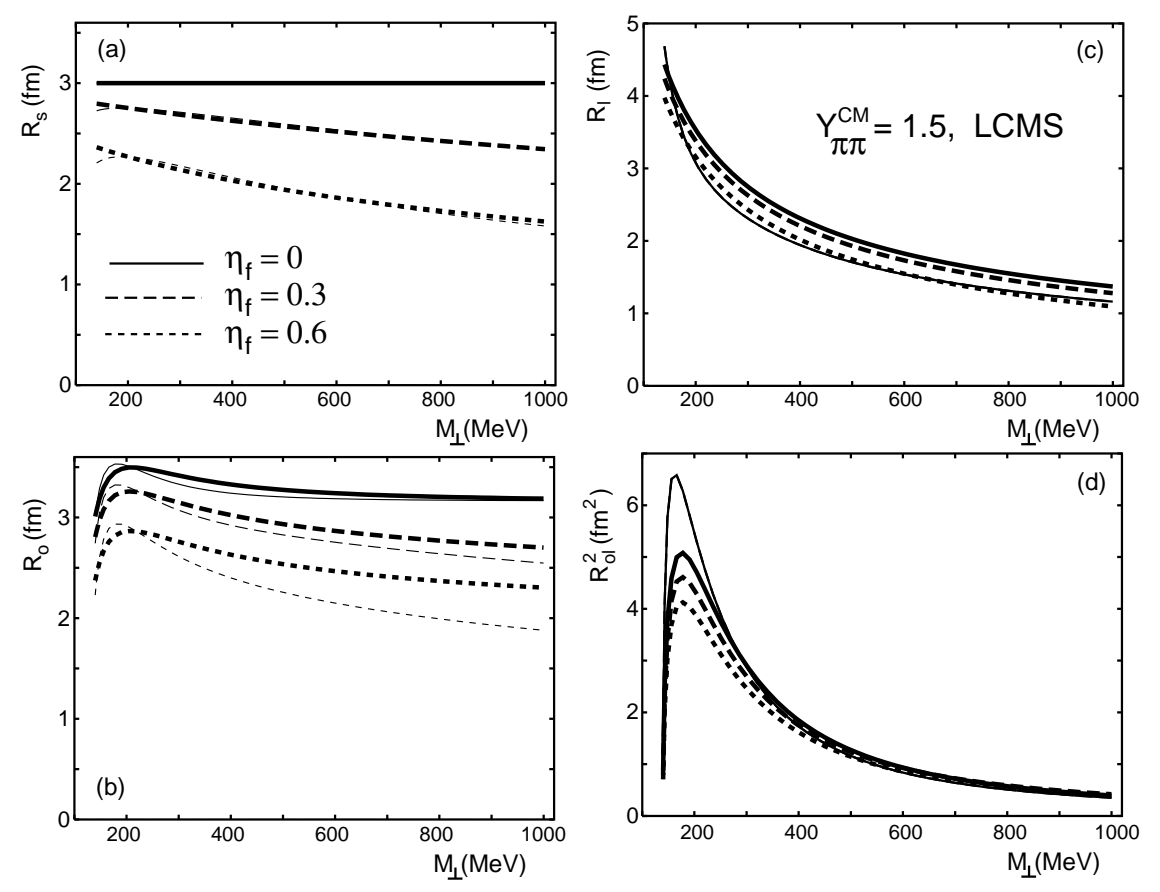

Fig.2. Same as Fig.1, but now evaluated in the LCMS. Please note the change of sign and magnitude of the cross-term. (Figure taken from Ref. [27].)

\section{MOMENTUM DEPENDENCE OF HBT PARAMETERS}

\subsection{Standard Cartesian fit}

In Fig. 1 I show the HBT radius parameters from the standard Cartesian fit (14) for pion pairs with c.m. rapidity $Y=1.5$ where the fit of the correlator is done in the CMS [27]. The different thick curves correspond to different strengths $\eta_{f}$ of the transverse flow. Without transverse flow $R_{s}$ is $M_{\perp}$-independent because the source (29) has no transverse temperature gradients. As transverse flow increases, $R_{s}$ develops an increasing dependence on $M_{\perp}$. It can be approximated by an inverse power law, with the power increasing monotonously with $\eta_{f}\left[12,14 . R_{l}\right.$ features a very strong $M_{\perp}$-dependence even without transverse flow, due to the strong longitudinal expansion of the source. It can 
also be described by an inverse power law, with a larger power $\simeq 0.55$, in rough agreement with the approximate $\sqrt{T / M_{\perp}}$-scaling law suggested in [2] (see, however, [ 12, 21] for a more quantitative discussion). The increase of $R_{o}$ at small $M_{\perp}$ is due to the contribution (19) from the effective lifetime. As seen in Fig. 4 below, in the YK frame (source rest frame) the latter is of order $2.5 \mathrm{fm} / c$ at small $M_{\perp}$; Fig. $1 \mathrm{~b}$ shows that its effect on $R_{o}$ compared to $R_{s}$ in the CMS is much smaller (and thus more difficult to measure). Fig. 1d shows that the cross-term is small in the CMS but non-zero. It vanishes at $K_{\perp}=0$ by symmetry and also becomes small again at large $K_{\perp}$.

The thin lines in Fig. 1 show for comparison approximate results for the HBT radii calculated from the approximate analytical results given in Ref. [5] which were derived by evaluating Eqs. (15) 18) by saddle point integration. It is clear that this method fails here (see Ref. [ 12] for a quantitative discussion of this approximation), and that the analytical expressions should not be used for a quantitative analysis of HBT data.
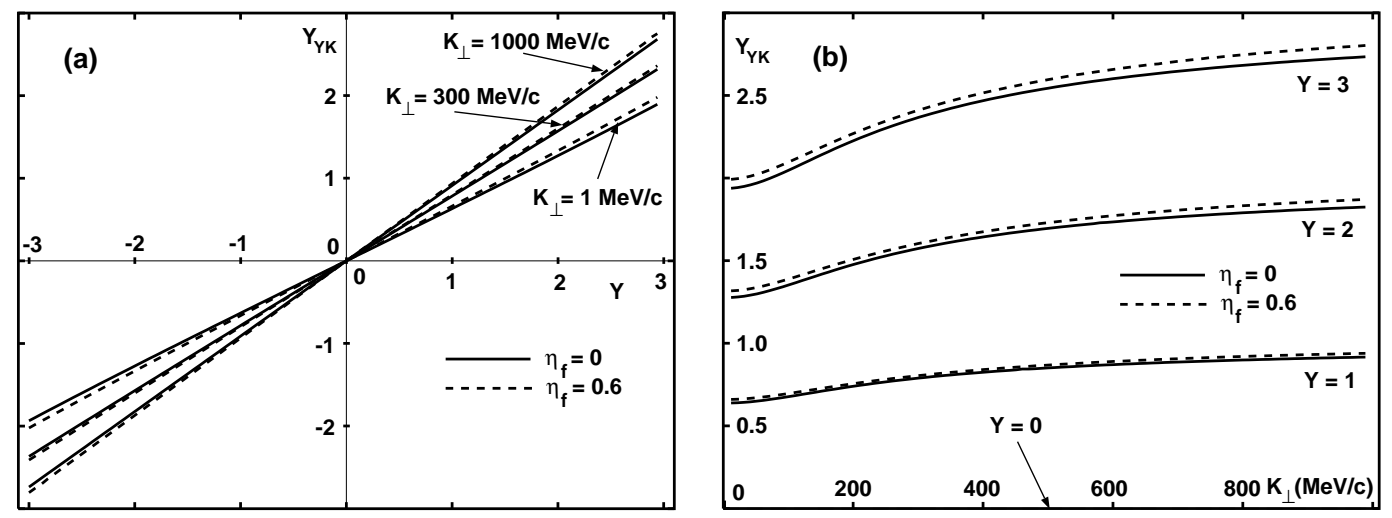

Fig.3. (a) The Yano-Koonin rapidity for pion pairs, as a function of the pair c.m. rapidity $Y$, for various values of $K_{\perp}$ and two values for the transverse flow $\eta_{f}$. (b) The same, but plotted against $K_{\perp}$ for various values of $Y$ and $\eta_{f}$. (Figure taken from Ref. [13].)

Fig. 2 shows the same situation as Fig. 1, but now all HBT radii are evaluated in the LCMS (longitudinally comoving system [ 22]) which moves with the pair rapidity $Y=1.5$ relative to the CMS. A comparison with Fig. 1 shows the strong reference frame dependence of the standard HBT radii. In particular, the cross-term changes sign and is now much larger. The analytical approximations from Ref. [5] work much better in the LCMS [5], but for $R_{o}$ and $R_{o l}^{2}$ they are still not accurate enough (in particular in view of the delicate nature of the lifetime effects on $R_{o}$ ).

\subsection{The Yano-Koonin velocity}

Fig. 3 shows (for pion pairs) the dependence of the YK velocity on the pair momentum K. In Fig. 3a we show the YK rapidity $Y_{\mathrm{YK}}=\frac{1}{2} \ln [(1+v) /(1-v)]$ as a function of the pair rapidity $Y$ (both relative to the CMS) for different values of $K_{\perp}$, in Fig. 3b the same quantity as a function of $K_{\perp}$ for different $Y$. Solid lines are without transverse flow, dashed lines are for $\eta_{f}=0.6$. For large $K_{\perp}$ pairs, the YK rest frame approaches the LCMS (which moves with the pair rapidity $Y$ ); in this limit all pairs are thus emitted from a small region 
in the source which moves with the same longitudinal velocity as the pair. For small $K_{\perp}$ the YK frame is considerably slower than the LCMS; this is due to the thermal smearing of the particle velocities in our source around the local fluid velocity $u^{\mu}(x)$ [ 14]. The linear relationship between the rapidity $Y_{\mathrm{YK}}$ of the Yano-Koonin frame and the pion pair rapidity $Y$ is a direct reflection of the boost-invariant longitudinal expansion flow [ 13]. For a non-expanding source $Y_{\mathrm{YK}}$ would be independent of $Y$. Additional transverse flow is seen to have nearly no effect. The dependence of the YK velocity on the pair rapidity thus measures directly the longitudinal expansion of the source and cleanly separates it from its transverse dynamics. A detailed discussion of these features is given in Ref. [14].

\section{3. $M_{\perp}$-scaling of $\mathrm{YKP}$ radii and transverse flow}

In the absence of transverse flow, a thermal source like (29) depends on the particle rest mass and on the transverse momentum $K_{\perp}$ only through the combination $M_{\perp}^{2}=$ $m^{2}+K_{\perp}^{2}$ (see Eq. (31)). Furthermore, the source is then azimuthally and $x \rightarrow-x$ reflection symmetric. Hence $\langle\tilde{x} \tilde{t}\rangle,\langle\tilde{x} \tilde{z}\rangle$, and $\left\langle\tilde{x}^{2}-\tilde{y}^{2}\right\rangle$ all vanish and the approximations in Eqs. (23,24) become exact. As a result, all three YKP radii (22)-(24) are only functions of $M_{\perp}$, too (as well as of $\mathrm{Y}$, of course), i.e. they do not depend explicitly on the particle rest mass.

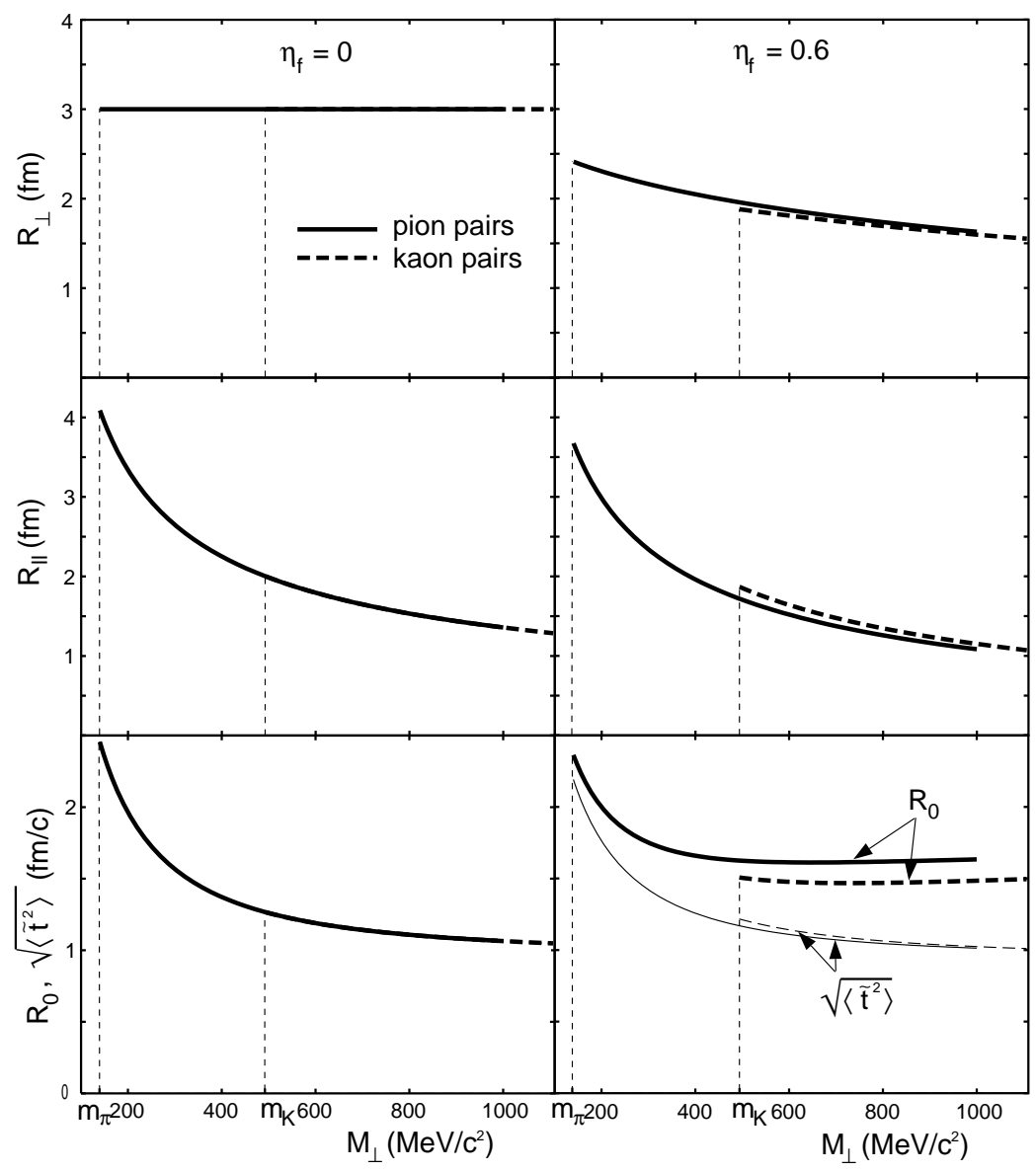


Fig.4. The YKP radii $R_{\perp}, R_{\|}$, and $R_{0}$ (from top to bottom) for vanishing transverse flow (left column) and for $\eta_{f}=0.6$ (right column), as functions of $M_{\perp}$ for pairs at $Y_{\mathrm{cm}}=0$. Solid (dashed) lines are for pions (kaons). The breaking of the $M_{\perp}$-scaling by transverse flow is obvious in the right column. Also, as shown in the lower right panel, for nonzero transverse flow $R_{0}$ does not agree exactly with the effective source lifetime $\sqrt{\left\langle\tilde{t}^{2}\right\rangle}$. (Figure taken from Ref. [14].)

This is seen in the left column of Fig. 4 where the three YKP radii are plotted for $Y_{\mathrm{cm}}=0$ pion and kaon pairs as functions of $M_{\perp}$; they agree perfectly. The transverse radius here shows no $M_{\perp}$-dependence due to the absence of transverse temperature gradients, but even with temperature gradients it would only depend on $M_{\perp}$. (Of course, this discussion neglects resonance decays which will be studied in Sec. 7.) Note that $M_{\perp^{-}}$ scaling in the absence of transverse flow applies only to the YKP radius parameters: since the expressions (16)-(18) involve nonvanishing variances with $\beta_{\perp^{-}}$or $\beta_{l}$-prefactors (which depend explicitly on the rest mass), the HBT radii from the standard Cartesian fit do not exhibit $M_{\perp}$-scaling.

For non-zero transverse flow $\eta_{f} \neq 0$ this $M_{\perp}$-scaling is broken by two effects: first, the second term in (31) destroys the $M_{\perp}$-scaling of the emission function itself, and second the $\boldsymbol{\beta}$-dependent correction terms in (23, 24) are now non-zero because the same term also breaks, for $K_{\perp} \neq 0$, the $x \rightarrow-x$ and $x \rightarrow y$ symmetries. The magnitude of the associated scale breaking due to the pion-kaon mass difference is seen in the right column of Fig. 4 for $\eta_{f}=0.6$. The effects are small and require very accurate experiments for their detection. However, the sign of the effect is opposite for $R_{\|}$and for $R_{\perp}, R_{0}$ which may help to distinguish flow-induced effects from resonance decay contributions.

Since for $Y_{\mathrm{cm}}=0$ the YK and CMS frames coincide, $\beta_{l}=0$ in the YK frame and the approximation in (23) remains exact even for non-zero transverse flow. The same is not true for the approximation in (24), and therefore we show in the lower right panel of Fig. 4 also the effective source lifetime $\sqrt{\left\langle\tilde{t}^{2}\right\rangle}$ for comparison. The apparently rather large discrepancies between the YKP parameter $R_{0}$ and the effective source lifetime is due to a rather extreme choice of parameters: a large flow transverse flow and a small intrinsic source lifetime of $\Delta \tau=1 \mathrm{fm} / c$ in (29). Since $\sqrt{\left\langle\tilde{t}^{2}\right\rangle}$ approaches $\Delta \tau$ in the limit of large $M_{\perp}$ while the dominant [14] correction term $\left\langle\tilde{x}^{2}-\tilde{y}^{2}\right\rangle$ does not depend on $\Delta \tau$, the YKP parameter $R_{0}$ will track the effective source lifetime more accurately for larger values of $\Delta \tau$ (and for smaller values of $\eta_{f}$ ).

Why do $\sqrt{\left\langle\tilde{t}^{2}\right\rangle}$ and $R_{0}$ increase at small $M_{\perp}$ ? Due to the rapid longitudinal expansion, the longitudinal region of homogeneity $R_{\|}$is a decreasing function $M_{\perp}$. Since for different pair momenta $R_{0}$ measures the source lifetime in different YK reference frames, the freeze-out "hypersurface" will in general appear to have different shapes for pairs with different momenta. Only in our model, where freeze-out occurs at fixed proper time $\tau_{0}$ (up to a Gaussian smearing with width $\Delta \tau$ ), is it frame-independent. It is thus generally unavoidable (and here, of course, true in any frame) that freeze-out at different points $z$ in the source will occur at different times $t$ in the YK frame. Since a $z$-region of size $R_{\|}$contributes to the correlation function, $R_{\|}$determines how large a domain of this freeze-out surface (and thus how large an interval of freeze-out times in the YK frame) is sampled 
by the correlator. This interval of freeze-out times combines with the intrinsic Gaussian width $\Delta \tau$ to yield the total effective duration of particle emission. It will be largest at small pair momenta where the homogeneity region $R_{\|}$is biggest, and will reduce to just the variance of the Gaussian proper time distribution at large pair momenta where the longitudinal (and transverse) homogeneity regions shrink to zero. The rise of $\Delta t(\mathbf{K})$ at small $\mathbf{K}$ is thus generic.

\section{RESONANCE DECAYS}

The proportionality of the $M_{\perp}$-dependence of $R_{\perp}$ to the transverse flow $\eta_{f}$ and the particular pattern of $M_{\perp}$ scale-breaking by the latter open an avenue for the quantitative extraction of transverse flow from HBT data [26]. This requires, however, that the $M_{\perp}$-dependence of $R_{\perp}$ is not affected by resonance decays. Since they contribute more to pions than to kaons they may also affect the $M_{\perp}$-scaling arguments. The work by the Marburg group [10] on resonance decay effects on HBT in the context of hydrodynamical simulations indicates, within the standard Cartesian framework and without accounting for the cross-term, a possible additional $M_{\perp}$-dependence of the transverse radius. However, a systematic analysis of resonance contributions to HBT as a function of various characteristic source parameters is only now becoming available [28].

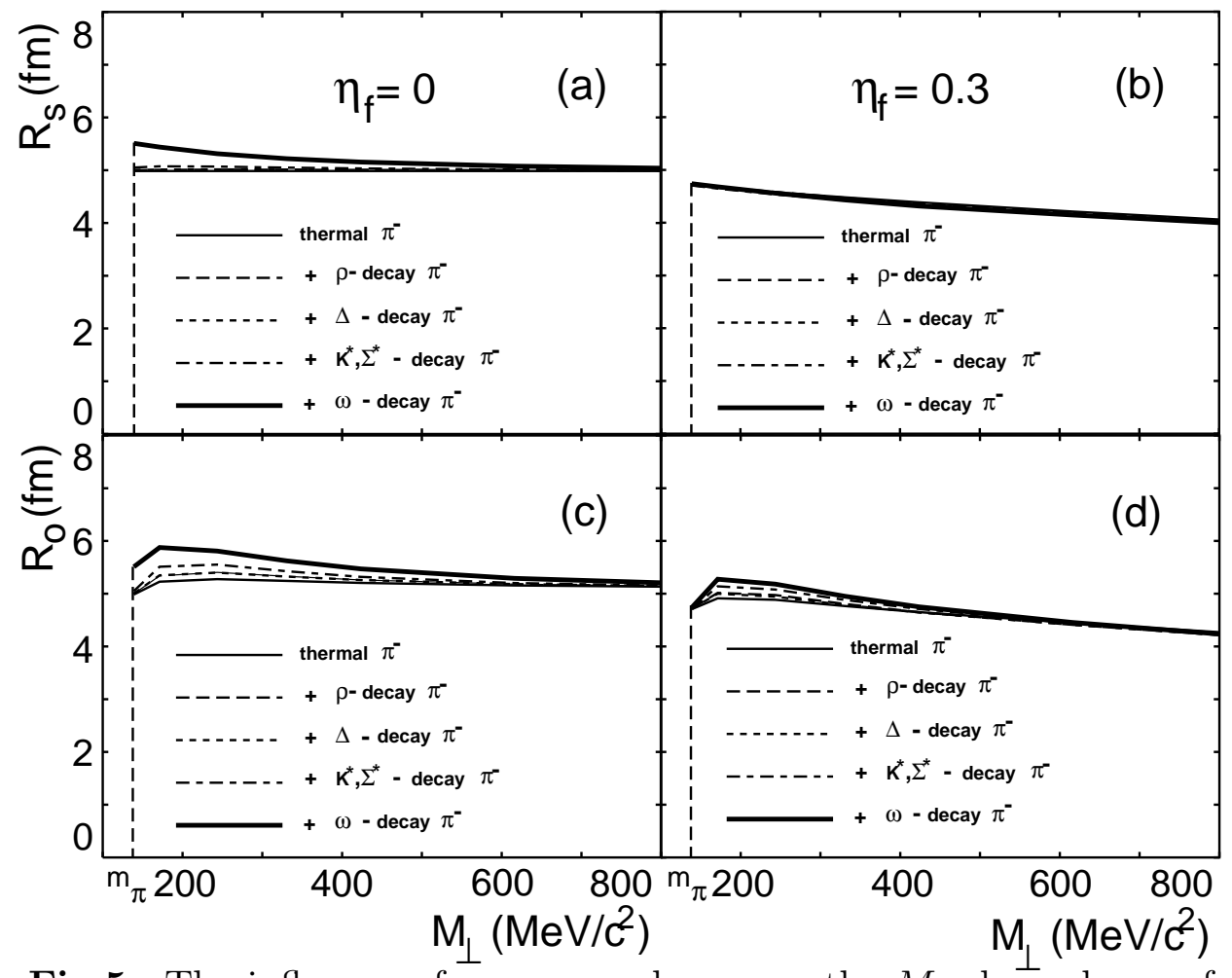

Fig.5. The influence of resonance decays on the $M_{\perp}$-dependence of $R_{s}$ (a,b) and $R_{o}(\mathrm{c}, \mathrm{d})$ for $Y_{\mathrm{cm}}=0$ pion pairs. a,c: no transverse flow; b,d: transverse flow rapidity $\eta_{f}=0.3$. The Gaussian transverse radius is here $R=5 \mathrm{fm}$, and $T=150 \mathrm{MeV}$. (Figure taken from Ref. [28].)

In Fig. 5 I show some results from Ref. [28] for the same emission function (29). The only change for resonances is an additional spin degeneracy factor and the different rest 
mass. The complete spectrum of relevant resonances is included, and in the decays the 2- and 3-body decay kinematics is fully taken into account. The HBT radii are extracted from a Gaussian fit to the numerically calculated correlation function. A detailed technical discussion is given in Ref. [28].

Fig. 5 shows that the effects of the short-lived resonances with lifetimes of order 1 $\mathrm{fm} / c$ on $R_{s}$ are essentially negligible, both at vanishing and at nonzero transverse flow. Only the $\omega$ with its intermediate lifetime of $20 \mathrm{fm} / c$ affects $R_{s}$, but only for vanishing transverse flow. There it induces a weak $M_{\perp}$-dependence at small $M_{\perp}$ even in the absence of transverse flow; at $M_{\perp}>500 \mathrm{MeV}$ the contribution of the $\omega$ dies out, and $R_{s}$ again becomes $M_{\perp}$-independent (which would not be the case if it were affected by flow). At $\eta_{f}=0.3$ and 0.6 [28] not even the $\omega$ generates any additional $M_{\perp}$-dependence! $-R_{o}$ shows some effects from the additional lifetime of the resonances, in particular from the long-lived $\omega$. Resonances with much longer lifetimes than the $\omega$ (in particular all weak decays) have no effect on the radii, because their contribution to the correlator is only at very small values of $q$ which cannot be resolved experimentally. They lead to a reduced "incoherence parameter" $\lambda\left[\right.$ [10, 15]. Since for increasing $M_{\perp}$ the resonance contributions decrease, the $\lambda$-parameter increases with $M_{\perp}$, approaching 1 as $M_{\perp} \rightarrow \infty$ [ 10, 15]. A detailed study will follow [28].

The weak effect of resonances on $R_{s}=R_{\perp}$ seems surprising: due to their non-zero lifetime they should be able to propagate outside the original source before decay and form a pion "halo" [10, 15]. This effect is, however, much weaker than naively expected: most of the resonances are not very fast, and the halo thickness is thus only a fraction of the resonance lifetime. At finite transverse flow an additional effect comes into play: it turns out that then the effective size of the emission function for directly emitted resonances is smaller than that for direct pions [28]! At $\eta_{f}=0.3$ and 0.6 this even slightly overcompensates the halo effect, and altogether the resonances change neither the size nor the $M_{\perp}$-dependence of $R_{s}$.

\section{CONCLUSIONS}

The model-independent expressions of Secs. 3 and 5 for the HBT width parameters in terms of second order variances of the emission function provide the basis of a detailed physical interpretation of the measured HBT radii. They show that the HBT radius parameters do not necessarily measure the full geometric extension of the source, but regions of homogeneity in the effective emission function for particles with certain fixed momenta. For expanding systems these are usually smaller than the naive geometric source size and decreasing functions of the pair momentum. For systems with finite lifetime the HBT parameters usually mix the spatial and temporal structure of the source, and their unfolding requires model studies.

With the new YKP parametrization we have found a method which, for systems with dominant longitudinal expansion, cleanly factorises the longitudinal and transverse spatial from the temporal homogeneity length. The effective source lifetime is directly fitted by the parameter $R_{0}$; it is generically a function of the pair momentum and largest for pairs which are slow in the CMS. Another fit parameter, the YK velocity, measures directly the longitudinal velocity of the emitting fluid element, and its dependence on the pair rapidity 
allows for a direct determination of the longitudinal expansion of the source. Without transverse expansion, the YKP radius parameters show exact $M_{\perp}$-scaling. The breaking of this scaling and the $M_{\perp}$-dependence of the transverse radius parameter $R_{\perp}$ allow for a determination of the transverse expansion velocity of the source. Resonance decays were shown to mostly affect the lifetime parameter and leave the $M_{\perp}$-dependence of $R_{\perp}$ nearly unchanged. They thus do not endanger the extraction of the transverse flow via HBT.

With this new and detailed understanding of the method, I believe that HBT interferometry has a begun a new and vigorous life as a powerful tool for reconstructing the geometric and dynamic space-time characteristics of the collision zone from the measured momentum spectra.

Acknowledgements: I thank my collaborators on this project, S. Chapman, J.R. Nix, B. Tomášik, U.A. Wiedemann, and Wu Yuanfang, who each contributed valuable pieces to the puzzle. Without their help until the very last minutes before the conference this review would have been impossible. I would also like to acknowledge fruitful discussions with H. Appelshäuser, T. Csörgő, D. Ferenc, M. Gaździcki, and P. Seyboth. This work was supported by grants from BMBF, DFG, and GSI.

\section{REFERENCES}

1. D. Boal, C.G. Gelbke, and B. Jennings, Rev. Mod. Phys. 62 (1990) 553.

2. A.N. Makhlin and Yu.M. Sinyukov, Z. Phys. C39 (1988) 69.

3. U. Mayer, E. Schnedermann, and U. Heinz, Phys. Lett. B294 (1992) 69.

4. S.V. Akkelin and Yu.M. Sinyukov, Phys. Lett. B356 (1995) 525.

5. T. Csörgö and B. Lörstad, Nucl. Phys. A590 (1995) 465c; and Lund University preprint LUNFD6/(NFFL-7082) hep-ph/9509213, Phys. Rev. C, in press.

6. S. Chapman, P. Scotto, and U. Heinz, Phys. Rev. Lett. 74 (1995) 4400; and Heavy Ion Physics 1 (1995) 1.

7. S. Pratt, Phys. Rev. Lett. 53 (1984) 1219; Phys. Rev. D33 (1986) 1314.

8. G. Bertsch, Nucl. Phys. A498 (1989) 173c.

9. S. Pratt, T. Csörgő, and J. Zimányi, Phys. Rev. C42 (1990) 2646.

10. B.R. Schlei et al., Phys. Lett. B293 (1992) 275; J. Bolz et al., Phys. Lett. B300 (1993) 404; and Phys. Rev. D47 (1993) 3860.

11. S. Chapman, J.R. Nix, and U. Heinz, Phys. Rev. C52 (1995) 2694.

12. U.A. Wiedemann, P. Scotto and U. Heinz, Phys. Rev. C53 (1996) 918.

13. U. Heinz, B. Tomášik, U.A. Wiedemann, and Wu Y.-F., Phys. Lett. B382 (1996) 181.

14. Y.-F. Wu, U. Heinz, B. Tomášik, and U.A. Wiedemann, submitted to Phys. Rev. C nucl-th/9607044.

15. T. Csörgö, B. Lörstad, and J. Zimányi, Z. Phys. C, in press [hep-ph/9411307).

16. For a discussion of Coulomb final state interactions see G. Baym and P. BraunMunzinger, nucl-th/9606055, and references therein, as well as G. Baym's contribution to this volume.

17. M. Gyulassy, S.K. Kauffmann, and L.W. Wilson, Phys. Rev. C20 (1979) 2267.

18. S. Chapman and U. Heinz, Phys. Lett. B340 (1994) 250.

19. E. Shuryak, Phys. Lett. B44 (1973) 387; Sov. J. Nucl. Phys. 18 (1974) 667. 
20. Wu Y.-F. and U. Heinz, submitted to Phys. Lett. B hucl-th/9607037).

21. M. Herrmann and G.F. Bertsch, Phys. Rev. C51 (1995) 328.

22. T. Csörgö and S. Pratt, in Proceedings of the Workshop on Relativistic Heavy Ion Physics at Present and Future Accelerators, Budapest, 1991, edited by T. Csörgö et al. (MTA KFKI Press, Budapest, 1991), p. 75.

23. D.H. Rischke and M. Gyulassy, Columbia preprint CU-TP-756 (nucl-th/9606039).

24. F. Yano and S. Koonin, Phys. Lett. B78 (1978) 556.

25. M.I. Podgoretskiu, Sov. J. Nucl. Phys. 37 (1983) 272.

26. U. Heinz, B. Tomášik, U.A. Wiedemann, and Wu Y.-F., Heavy Ion Physics (1996), in press nucl-th/9606041.

27. B. Tomášik and U. Heinz, TPR-96-16, and poster at QM96.

28. U.A. Wiedemann and U. Heinz, TPR-96-14, and poster at QM96. 